\title{
Laser polarization-variable autofluorescence of the network of optically anisotropic biological tissues: Diagnostics and differentiation of early stages of cancer of cervix uteri
}

\author{
Yu. A. Ushenko*, M. I. Sidor*, N. Pashkovskaia ${ }^{\dagger}$, \\ G. D. Koval ${ }^{\dagger}$, Yu. F. Marchuk ${ }^{\dagger}$ and D. Andreichuk ${ }^{\dagger}$ \\ ${ }^{*}$ Correlation Optics Department, Chernivtsi National University \\ 2 Kotsyubinsky Street, Chernivtsi 58012, Ukraine \\ ${ }^{\dagger}$ Clinical Immunology, Allergology and Endocrinology Department \\ Bucovinian State Medical University, 2 Theatral Sq. \\ Chernivtsi 58002, Ukraine \\ łyuriyu@gmail.com
}

Received 15 October 2013

Accepted 13 December 2013

Published 27 January 2014

\begin{abstract}
This research presents the results of investigation of laser polarization fluorescence of biological layers (histological sections, cytological smears) in the task of diagnostics and differentiation of early stages of cancer: Dysplasia - cervical microinvasive carcinoma of cervix uteri. The analytical conditions of polarization-optimal probing of biological layers were determined basing on the model of linear birefringence and dichroism of birefringent (fibrillar, porphyrin) networks. The technique of polarization-variable laser autofluorescence was developed and experimentally tested. The objective criteria (statistical moments) of differentiation of histological sections autofluorescent images of endometrium biopsy and cytological smears of it mucous coat were defined. The operational characteristics (sensitivity, specificity, accuracy) of this technique were determined concerning the positions of probative medicine, and clinical efficiency.
\end{abstract}

Keywords: Autofluorescence; polarization; birefringence; statistical optics; diagnostics; medical and autofluorescence imaging.

\section{$\$$ Corresponding author.}

This is an Open Access article published by World Scientific Publishing Company. It is distributed under the terms of the Creative Commons Attribution 3.0 (CC-BY) License. Further distribution of this work is permitted, provided the original work is properly cited. 


\section{Introduction}

Biological tissues are structurally inhomogeneous optically anisotropic media with absorption. The Mueller-matrix formalism is used for the most complete and unified description of polarization properties of such objects. ${ }^{1-6}$ Nowadays in biological and medical research, many practical techniques, based on the measurement and analysis of Mueller matrices of the samples, are under study. ${ }^{7,8}$ During the last 10-15 years, an independent direction - laser polarimetry — was formed.$^{9-11}$ On its basis, the interrelations between the set of statistical moments of the first-fourth-order characterizing the distributions of Mueller-matrix elements and the parameters of linear birefringence of fibrillar protein networks of human biological tissues were determined. Grounding on this, the diagnostics of pathological changes of skin derma, epithelial, and connective tissues of woman's reproductive sphere organs, etc. was realized. ${ }^{12-17}$

The optical techniques of investigation of biological tissues called "optical biopsy" are widespread in modern medical diagnostics. The analysis of luminescence of such objects occupies a special place among the optical biopsy techniques. One of the most attractive directions is the intrinsic fluorescence or autofluorescence.

The applied-physics fundamentals of laser radiation usage in the tasks of diagnostics of typical cancer processes by laser-induced fluorescence spectroscopy are developed. ${ }^{18-24}$

The next step in development of methods of biological tissues optical diagnostics consists in successful combining of polarimetric and fluorescent methods. ${ }^{25,26}$ The topical task in this direction is elaborating of new complex approaches concerning early diagnostics of pretumor changes of cervix uteri, which is the most widespread disease of women reproductive system. At that moment, the traditional technique of diagnostics of cervix uteri cancer consists of the following operations: "cytological screening" — "colposcopy analysis" - "biopsy". Each of these stages is not perfect. For the first two operations, the low specificity $(\sim 50 \%)$ is typical which leads to the groundless biopsy. The biopsy implementation leads to traumatization, local inflammatory reaction, and to the risk of development of cervix uteri cancer.
This research is focused on the development of polarization-variable technique of laser autofluorescence of cervix uteri tissues in the task of objectification of diagnostics and differentiation of early stages of cervix uteri cancer.

\section{Theory}

The model ideas of optical anisotropy of optically thin (attenuation coefficient $\tau<0.1$ ) biological layers (histological sections, smears) developed in the series of research works ${ }^{9-11,27}$ served as the basis for elaboration of polarization-variable laser autofluorescence technique. In this research, we shall present only a concise overview of basic concepts of this model necessary for understanding the materials of this particular article.

The polarization properties of optically anisotropic component of optically thin, transforming polarization (non-depolarizing) biological layer are describe by the generalized Mueller matrix $\{M\}$

$$
\{M\}=\{\Psi\}\{D\}=\left\|\begin{array}{cccc}
1 & M_{12} & M_{13} & M_{14} \\
M_{21} & M_{22} & M_{23} & M_{24} \\
M_{31} & M_{32} & M_{33} & M_{34} \\
M_{41} & M_{42} & M_{43} & M_{44}
\end{array}\right\| .
$$

Here $\{D\}-$ matrix of linear birefringence; $\{\Psi\}-$ matrix of linear dichroism.

In the case of birefringent structures (polypeptide chains, protein fibrils, liquid-crystal chains of porphyrins), the full form of the above mentioned matrix operators can be presented as follows ${ }^{9,26}$ :

$$
\begin{aligned}
& \{D\}=\left\|\begin{array}{cccc}
1 & 0 & 0 & 0 \\
0 & d_{22} & d_{23} & d_{24} \\
0 & d_{32} & d_{33} & d_{34} \\
0 & d_{42} & d_{43} & d_{44}
\end{array}\right\|, \\
& d_{i k}=\left\{\begin{array}{l}
d_{22}=\cos ^{2} 2 \rho+\sin ^{2} 2 \rho \cos \delta ; \\
d_{23}=d_{32}=\cos 2 \rho \sin 2 \rho(1-\cos \delta) ; \\
d_{33}=\sin ^{2} 2 \rho+\cos ^{2} 2 \rho \cos \delta ; \\
d_{24}=-d_{42}=\sin 2 \rho \sin \delta ; \\
d_{34}=-d_{43}=\cos 2 \rho \sin \delta ; \\
d_{44}=\cos \delta,
\end{array}\right. \\
& \{\Psi\}=\left\|\begin{array}{cccc}
1 & \varphi_{12} & \varphi_{13} & 0 \\
\varphi_{21} & \varphi_{22} & \varphi_{23} & 0 \\
\varphi_{31} & \varphi_{32} & \varphi_{33} & 0 \\
0 & 0 & 0 & \varphi_{44}
\end{array}\right\|,
\end{aligned}
$$




$$
\varphi_{i k}=\left\{\begin{array}{l}
\varphi_{12}=\varphi_{21}=(1-\Delta \tau) \cos 2 \rho \\
\varphi_{13}=\varphi_{31}=(1-\Delta \tau) \sin 2 \rho \\
\varphi_{22}=(1+\Delta \tau) \cos ^{2} 2 \rho+2 \sqrt{\Delta \tau} \sin ^{2} 2 \rho \\
\varphi_{23}=\varphi_{32}=(1-\Delta \tau) \sin 2 \rho \\
\varphi_{33}=(1+\Delta \tau) \sin ^{2} 2 \rho+2 \sqrt{\Delta \tau} \cos ^{2} 2 \rho \\
\varphi_{44}=2 \sqrt{\Delta \tau}
\end{array}\right.
$$

where $\Delta \tau=\frac{\tau_{x}}{\tau_{y}}, \quad\left\{\begin{array}{l}\tau_{x}=\tau \cos \rho ; \\ \tau_{y}=\tau \sin \rho\end{array}, \tau_{x}, \tau_{y}\right.$ - absorption coefficients of linearly polarized orthogonal components of the laser beam amplitude, $\rho$ - direction of optical axis, $\delta$ - phase shift between linearly polarized orthogonal components of the amplitude of autofluorescent radiation.

The processes of formation of polarization structure of such radiation of biological layer are described by the following vector-parametric equation

$$
S^{*}=\{M\} S_{0} .
$$

Here $S_{0}$ and $S^{*}$ - Stokes vectors, ${ }^{3,4,9}$ which completely determine the polarization azimuth $\alpha$ and ellipticity $\beta$ of illuminating and autofluorescent radiation.

We shall show only the situation of probing the biological tissue by linearly polarized $\left(\alpha_{0}\right)$ laser beam (that does not make our analysis incomplete). Thus considering (1), (2) Stokes vector $S^{*}$ (4) we write in the full form

$$
\begin{aligned}
S^{*}= & \left(\begin{array}{c}
1 \\
\cos 2 \alpha^{*} \cos 2 \beta^{*} \\
\sin 2 \alpha^{*} \cos 2 \beta^{*} \\
\sin 2 \beta^{*}
\end{array}\right)=\left(1+M_{12}+M_{13}\right)^{-1} \\
& \times\left(\begin{array}{c}
M_{21}+M_{22} \cos 2 \alpha_{0}+M_{23} \sin 2 \alpha_{0} \\
M_{31}+M_{32} \cos 2 \alpha_{0}+M_{33} \sin 2 \alpha_{0} \\
M_{41}+M_{42} \cos 2 \alpha_{0}+M_{43} \sin 2 \alpha_{0}
\end{array}\right) .
\end{aligned}
$$

From (5), the polarization characteristics of autofluorescent radiation are determined as functions of optical properties of biological layer $\left(M_{i k}\right)$ and polarization state $\left(\alpha_{0}\right)$ of the probing beam

$\alpha^{*}=0.5 \arctan \left(\frac{M_{31}+M_{32} \cos 2 \alpha_{0}+M_{33} \sin 2 \alpha_{0}}{M_{21}+M_{22} \cos 2 \alpha_{0}+M_{23} \sin 2 \alpha_{0}}\right) ;$

$$
\beta^{*}=0.5 \arcsin \left(\frac{M_{41}+M_{42} \cos 2 \alpha_{0}+M_{43}}{1+M_{12}+M_{13}}\right) .
$$

The analysis (2), (3), (6), (7) shows that by varying the polarization azimuth $\alpha_{0}$, the maximal absorption $(\Delta \tau=1)$ of probing beam can be reached and, therefore, the maximal intensity of autofluorescence of biological layer can be realized. The following analytical equation corresponds to this effect

$$
\bar{\alpha}_{0}=\rho \rightarrow \Delta \tau=1 .
$$

The minimal ellipticity $\left(\beta_{\min }^{*} \rightarrow 0\right)$ of polarization (7) of autofluorescent radiation appears to be the indicator of realization (8)

$$
\beta_{\text {min }}^{*}=0.5 \arcsin \left(M_{42} \cos 2 \bar{\alpha}_{0}+M_{43} \sin 2 \bar{\alpha}_{0}\right) \rightarrow 0 .
$$

From (9) we determine

$$
\bar{\alpha}_{0}=0.5 \arctan \left(\frac{M_{42}}{M_{43}}\right) .
$$

For real biological layer, the model Eqs. (8)-(10) obtain the probability sense, while the specific, statistically averaged extreme values of $\bar{\alpha}_{0}$ and $\beta_{\text {min }}^{*}$ are determined by the peculiarities of distribution of parameters $\rho$ and $\delta$.

Grounding on the stated model, we have considered the fundamental and applied aspects.

The fundamental one is connected with experimental approbation of polarization-variable laser autofluorescence in the task of obtaining the biological layers autofluorescent images with maximal intensity.

The applied one is involved in the following:

- the increasing of the sensitivity, performance, and objectivity of "fluorescent biopsy", which provides the visualization, of invisible for histology, small $(<1 \mathrm{~mm})$ tumors;

- the increasing of the specificity of "fluorescent cytology" as the primary stage of clinical screening.

Thus, the technique of polarization-variable laser autofluorescence is based on the two-stage investigation "cytology-biopsy" of cervix uteri tissues and consists of the following stages:

(1) the measurement of Mueller matrix $\{M\}$ characterizing the biological layers - histological section of cervix uteri biopsy and smears of their mucous coat; 
Yu. A. Ushenko et al.

(2) the determination of the optimal value of polarization azimuth $\bar{\alpha}_{0} \rightarrow \beta_{\min }^{*}$ (relation (10));

(3) the illumination of the sample of biological tissue by plane-polarized $\left(\bar{\alpha}_{0}\right)$ laser beam;

(4) the measurement of the intensity distribution $\left(I\left(\bar{\alpha}_{0}\right)\right)$ in the plane of autofluorescent image.

(5) the statistic analysis of intensity distribution of autofluorescent image.

(6) the determination of operational (sensitivity $S e$, specificity $S p$ and accuracy $A c$ ) characteristics of the method.

\section{Analysis and Discussion of Experimental Data}

The patients were separated on two groups:

- dysplasia (precancer state) — group 1 samples);

- cervical microinvasive carcinoma - group 2 (18 samples).

Histological sections of biopsy of cervix uteri endometrium were prepared according to standard technique on the freezing microtome. The samples obtaining for the cytology were performed after lavage of cervix uteri by $0.9 \%$ solution of $\mathrm{Na}-\mathrm{Cl}$. By means of Volkmann's curette, the scrapes from the cervix uteri were performed. The obtained material was applied on the glass substrate.

Experimental measurements were performed in the set of Stokes polarimeter ${ }^{9}$ with the use of spectrally selective band-pass light filters (Fig. 1).

To excite autofluorescence, we used the "blue" solid-state laser with wavelength $\lambda=0.412 \mu \mathrm{m}$ and variable power $W$ (in this research we used $W=50 \mathrm{~mW}$ ). A polarization illuminator consists of quarter-wave plates 3 ; 5 (achromatic true zeroorder waveplate) and polarizer 4 . The image of the biological layers 6 by means of polarization microscope objective 7 (Nikon CFI Achromat P, focal distance $-30 \mathrm{~mm}$, numerical aperture - 0.1 , magnification $-4 \times$ ) was projected in the plane of light-sensitive CCD-camera 11 (the Imaging Source DMK 41AU02.AS, monochrome 1/2" CCD, Sony ICX205AL (progressive scan), overall amount of pixels $-m \times n=1280 \times 960$, light sensitive area size $-7600 \times 6200 \mu \mathrm{m}$, sensitivity - $0.05 \mathrm{l} \times$, dynamic range $-8 \mathrm{bit}$, deviation of photosensitive characteristics from linear no more then $12 \%$ ). Polarization analysis of coordinate distribution of intensity of samples images 6 was carried out by means of quarterwave plate 8 and polarizer 9 .

The calculation of Mueller-matrix elements of the investigated samples was performed according to the following algorithm ${ }^{9}$ :

$$
\begin{array}{ll}
M_{11}=0.5\left(S_{1}^{0}+S_{1}^{90}\right) ; & M_{21}=0.5\left(S_{2}^{0}+S_{2}^{90}\right) ; \\
M_{12}=0.5\left(S_{1}^{0}-S_{1}^{90}\right) ; & M_{22}=0.5\left(S_{2}^{0}-S_{2}^{90}\right) ; \\
M_{13}=S_{1}^{45}-M_{11} ; & M_{23}=S_{2}^{45}-M_{21} ; \\
M_{14}=S_{1}^{\otimes}-M_{11} ; & M_{24}=S_{2}^{\otimes}-M_{21} ; \\
M_{31}=0.5\left(S_{3}^{0}+S_{3}^{90}\right) ; & M_{41}=0.5\left(S_{4}^{0}+S_{4}^{90}\right) ; \\
M_{32}=0.5\left(S_{3}^{0}-S_{3}^{90}\right) ; & M_{42}=0.5\left(S_{4}^{0}-S_{4}^{90}\right) ; \\
M_{33}=S_{3}^{45}-M_{31} ; & M_{43}=S_{4}^{45}-M_{41} ; \\
M_{34}=S_{3}^{\otimes}-M_{31} ; & M_{44}=S_{4}^{\otimes}-M_{41} .
\end{array}
$$

Here $S_{i=2 ; 3 ; 4}^{0 ; 45 ; 90 ; \otimes}$ - Stokes vector parameters in the points of digital image of histological section 6 , measured for the series of linearly $\left(0^{\circ} ; 45^{\circ} ; 90^{\circ}\right)$ and right-circularly $(\otimes)$ polarized probing laser beams.

In this work, we have focused on consideration of spectral-selective range - luminescence of liquidcrystal networks of porphyrins ${ }^{24}$ of biological layers in the red part of spectrum $\left(\lambda_{f}=0.63-0.65 \mu \mathrm{m}\right)$.

Plane-polarized laser beams with polarization azimuths $\alpha_{0}=0^{\circ} ; \bar{\alpha}_{0} ; 90^{\circ}$ were used as probing

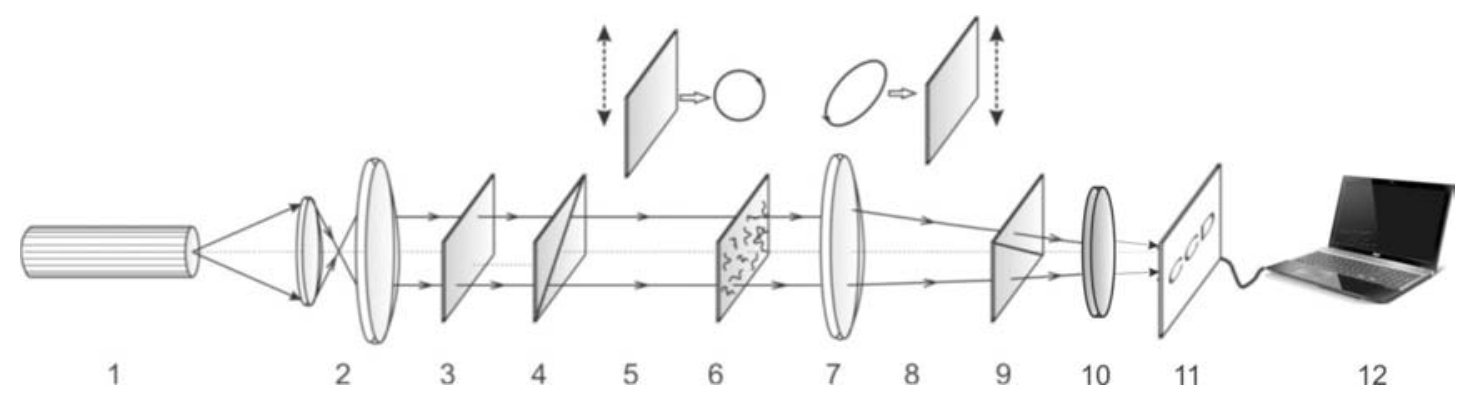

Fig. 1. Optical scheme of Stokes-polarimeter. Explanations are in the text. 
radiation exciting laser fluorescence of protein molecules. For each of such beams, the coordinate distributions of autofluorescence intensity $(I(m \times n))$ were measured using the band-pass $\left(\lambda_{\max }=\right.$ $632 \mu \mathrm{m})$ interference light filter 10 .

The objective assessment of distributions $I(m \times$ $n$ ) was performed by calculating the set ensemble of statistical moments of the first-fourth-orders which characterize its mean $\left(Z_{1}\right)$, dispersion $\left(Z_{2}\right)$, skewness $\left(Z_{3}\right)$ and kurtosis $\left(Z_{4}\right)$

$$
\begin{aligned}
& Z_{1}=\frac{1}{P} \sum_{j=1}^{P} I_{j} ; \quad Z_{2}=\sqrt{\frac{1}{P} \sum_{j=1}^{P}\left(I-Z_{1}\right)_{j}^{2}} ; \\
& Z_{3}=\frac{1}{Z_{2}^{3}} \frac{1}{P} \sum_{j=1}^{P}(I)_{j}^{3} ; \quad Z_{4}=\frac{1}{Z_{2}^{4}} \frac{1}{P} \sum_{j=1}^{P}(I)_{j}^{4},
\end{aligned}
$$

where $P$ is the number of pixels of CCD-camera.

The potentiality of the technique of polarization laser fluorescence is illustrated by the data presented in Figs. 2 and 3. Here the $I(m \times n)$ (fragments (1), (3), (5)) and histograms $N(I)$ (fragments (2), (4), (6)) of such distributions obtained for different states of polarization of the probing laser beam are presented.

The analysis of data presented in Figs. 2 and 3 proved the fundamental aspect of our model - the dependence of laser autofluorescence intensity (fragments (1), (3), (5)) of porphyrin molecules of endometrium on polarization state of the probing laser beam. It is obvious that for each value of $\alpha_{0}$ the extreme location and half-width of the histograms $N(I)$ are specific (fragments (2), (4), (6)). For optimal polarization state $\bar{\alpha}_{0}$, calculated according to (10), the shift of main extremes and half-width of histograms $N(I)$ into the domain of greater values of autofluorescence intensity reaches its maximal level (fragment (6)). This scenario is the most vividly observed for the autofluorescent images of the histological section of biopsy of cervix uteri endometrium with cervical microinvasive carcinoma.

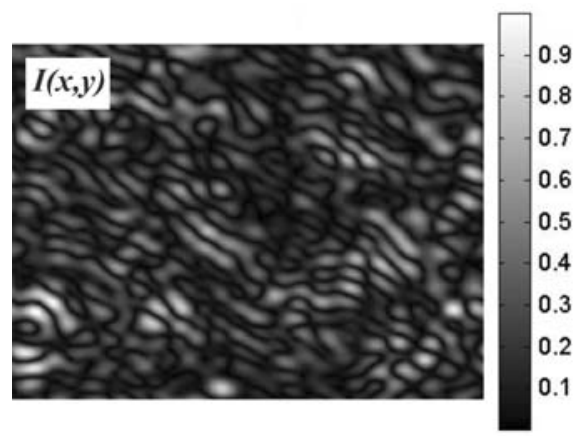

(a)

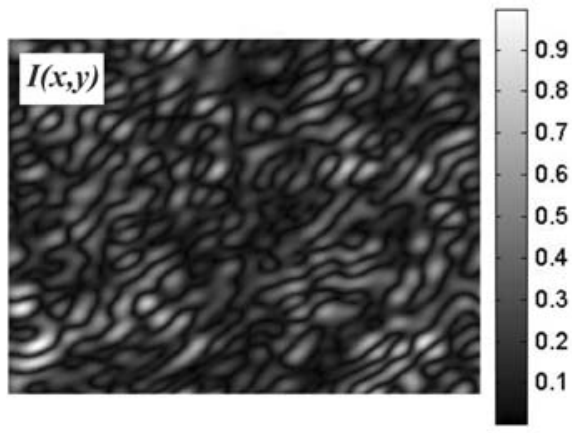

(c)

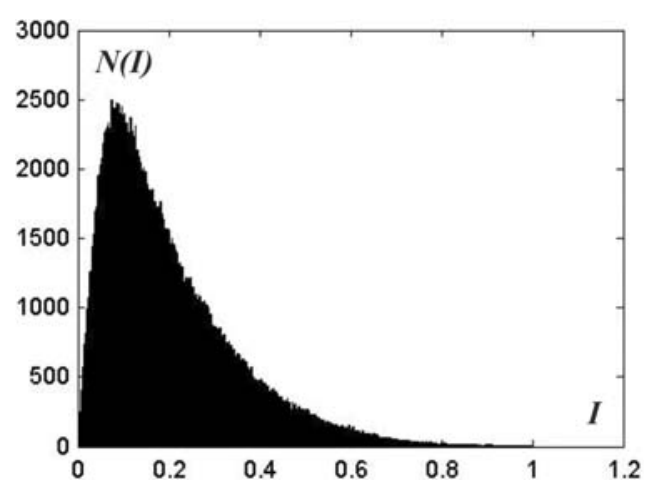

(b)

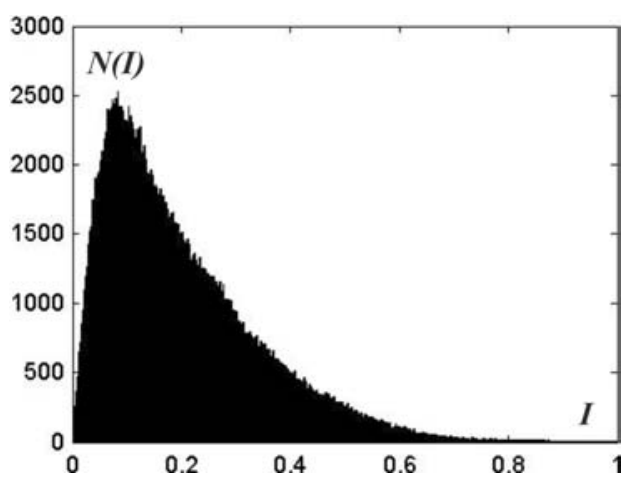

(d)

Fig. 2. Coordinate $(I(x, y))$ and statistical $(N(I))$ distributions of laser autofluorescence intensities of histological section of biopsy of cervix uteri endometrium in precancer state (dysplasia) for the following polarization states: $\alpha_{0}=0^{\circ}-$ fragments $(1)$, (2); $\alpha_{0}=90^{\circ}$ - fragments (3), (4); $\alpha_{0}=\bar{\alpha}_{0}$ - fragments (5), (6). Explanations are in the text. 


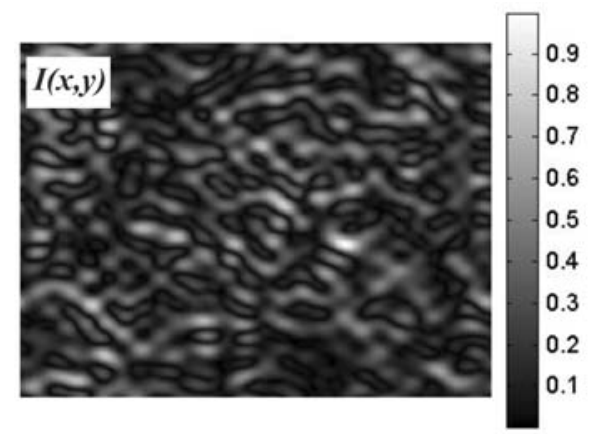

(e)

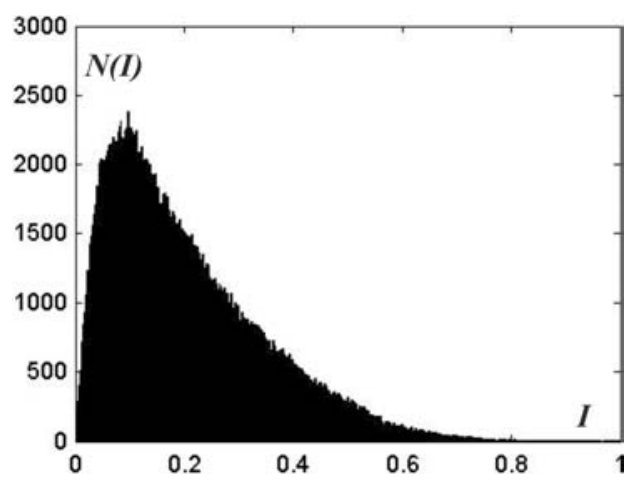

(f)

Fig. 2. (Continued)

Let us analyze the obtained results from the physical point of view. According to the considered model ideas, one determinate direction of a fibril defining the optimal polarization azimuth $\left(\bar{\alpha}_{0}\right)$ of the probing beam is realized in the plane of the biological tissue layer. Statistically, the distribution of fibrils directions of such a virtual object appears

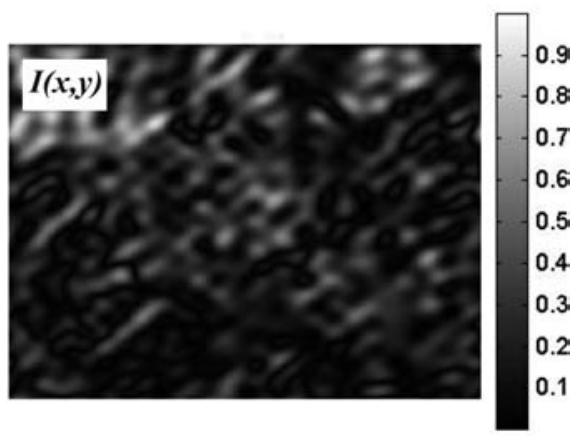

(a)

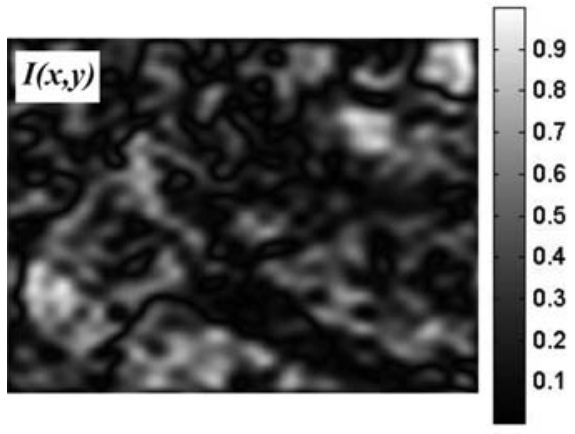

(c) to be a $\delta$-function. In the plane of histological sections of the samples of real biological tissues, a wide range of the directions of protein fibrils packing is realized. The more equiprobable in directions the fibrillar network is, the lesser the autofluorescence polarization dependence is, and vice versa. The fibrillar network of endometrium with cervical

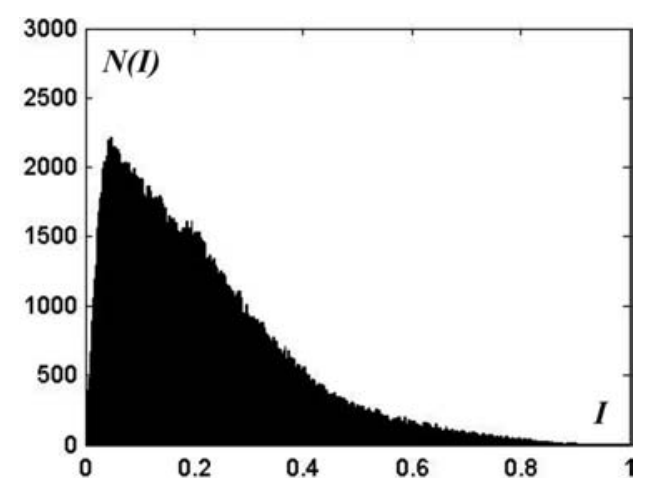

(b)

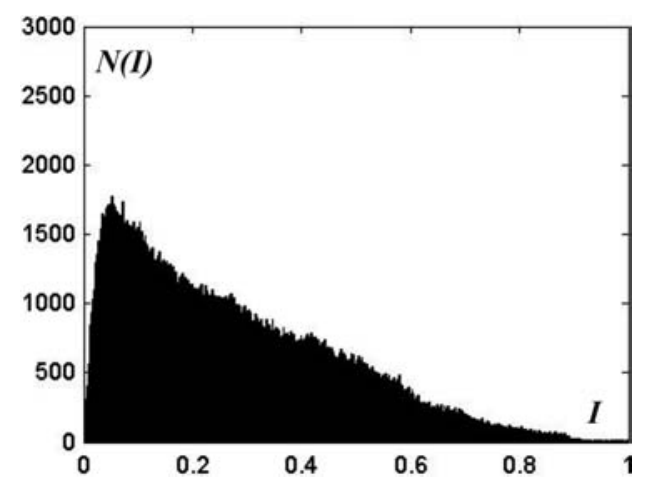

(d)

Fig. 3. Coordinate $(I(x, y))$ and statistical $(N(I))$ distributions of laser autofluorescence intensities of histological section of biopsy of cervix uteri endometrium (cervical microinvasive carcinoma) for the following polarization states: $\alpha_{0}=0^{\circ}-$ fragments $(1)$, (2); $\alpha_{0}=90^{\circ}$ - fragments (3), (4); $\alpha_{0}=\bar{\alpha}_{0}$ - fragments (5), (6). Explanations are in the text. 


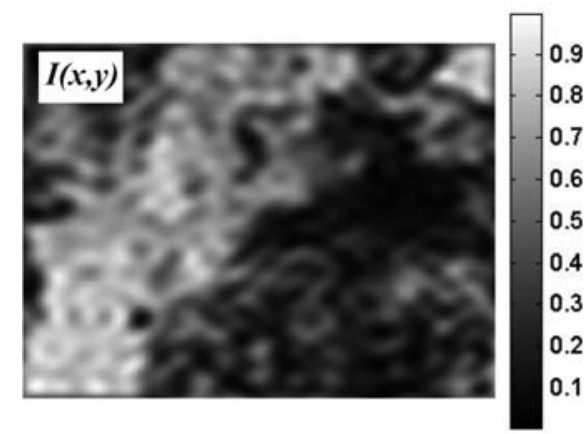

(e)

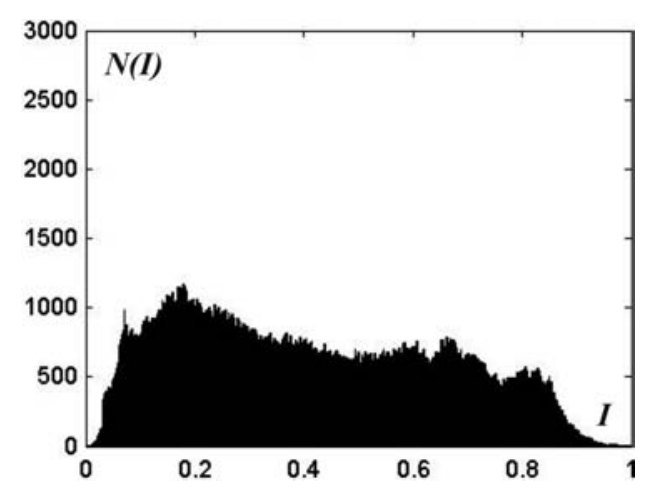

(f)

Fig. 3. (Continued)

microinvasive carcinoma is more determinate in directions. This fact is related to the directions of fibrillar growth of malignant tumor. As a result, a certain statistically prevailing direction $\rho^{*}$ of such growth is formed. It determines greater intensity of polarization autofluorescence of histological section of adenocarcinoma in comparison with the sample of endometrium with dysplasia (Figs. 2 and 3 , fragment (6)).

The applied aspect of "fluorescent biopsy" are quantitatively detected by the following variations of statistical moments $Z_{i=1 ; 2 ; 3 ; 4}(I)$ values:

- the mean $Z_{i=1}(I)$ of histogram $N(I)$ increases $(\uparrow)$;

- the dispersion $Z_{i=2}(I) \uparrow$ of distribution $N(I)$ increases;

- the skewness $Z_{i=3}(I) \uparrow$ of distribution $N(I)$ increases;

- the kurtosis of $N(I)$ determined by excess of $Z_{i=4}$ $(I) \downarrow$ decreases.
Tables 1 and 2 presents the averaged values and standard deviations of statistical moments $Z_{i=1 ; 2 ; 3 ; 4}(I)$ and operational characteristics of such a method.

The following quantitative (objective) criteria of diagnostics and differentiation of early stages types of cervix uteri cancer are determined:

- the difference between the values of statistical moments of the firsr-fourth-orders characterizing the distributions $I\left(\alpha_{0}\right)$ is for $Z_{1} \uparrow$ (by 1.09-1.06 times), for $Z_{2} \uparrow$ (by 1.12-1.53 times), for $Z_{3} \uparrow$ (by $1.2-1.63$ times) and for $Z_{4} \downarrow$ (by 1.16-1.49 times);

- the statistical distributions of $N(I)$ are the most vividly differentiated at probing by polarizationoptimal $\left(\bar{\alpha}_{0}\right)$ laser beam $-Z_{1} \uparrow$ (by 2.62 times), $Z_{2} \uparrow$ (by 1.71 times), $Z_{3} \uparrow$ (by 2.38 times) and $Z_{4} \downarrow$ (by 3.25 times).

Thus the method of statistical analysis of intensity distributions of laser polarization-variable

Table 1. Statistical $\left(Z_{i=1 ; 2 ; 34}\right)$ parameters of distributions $I\left(\alpha_{0}\right)$ of histological sections of biopsy of cervix uteri endometrium of Group 1 (dysplasia) and Group 2 (cervical microinvasive carcinoma).

\begin{tabular}{|c|c|c|c|c|c|c|}
\hline \multirow[b]{2}{*}{$Z_{i=1 ; 2 ; 3 ; 4}, \Delta Z_{i=1 ; 2 ; 3 ; 4}$} & \multicolumn{2}{|c|}{$I\left(\alpha_{0}=0^{\circ}\right)$} & \multicolumn{2}{|c|}{$I\left(\alpha_{0}=90^{\circ}\right)$} & \multicolumn{2}{|c|}{$I\left(\bar{\alpha}_{0}\right)$} \\
\hline & Group 1 & Group 2 & Group 1 & Group 2 & Group 1 & Group 2 \\
\hline$Z_{1}$ & $0.11 \pm 0.016$ & $0.12 \pm 0.019$ & $0.15 \pm 0.023$ & $0.24 \pm 0.028$ & $0.16 \pm 0.021$ & $0.42 \pm 0.058$ \\
\hline$\Delta Z_{1}$ & \multicolumn{2}{|c|}{1.09} & \multicolumn{2}{|c|}{1.6} & \multicolumn{2}{|c|}{2.62} \\
\hline$Z_{2}$ & $0.15 \pm 0.023$ & $0.23 \pm 0.029$ & $0.28 \pm 0.036$ & $0.33 \pm 0.041$ & $0.21 \pm 0.029$ & $0.36 \pm 0.041$ \\
\hline$\Delta Z_{2}$ & \multicolumn{2}{|c|}{1.53} & \multicolumn{2}{|c|}{1.12} & \multicolumn{2}{|c|}{1.71} \\
\hline$Z_{3}$ & $0.69 \pm 0.088$ & $0.83 \pm 0.094$ & $0.71 \pm 0.094$ & $1.16 \pm 0.18$ & $0.53 \pm 0.087$ & $1.26 \pm 0.18$ \\
\hline$\Delta Z_{3}$ & \multicolumn{2}{|c|}{1.2} & \multicolumn{2}{|c|}{1.63} & \multicolumn{2}{|c|}{2.38} \\
\hline$Z_{4}$ & $1.21 \pm 0.19$ & $0.81 \pm 0.097$ & $1.03 \pm 0.14$ & $0.89 \pm 0.097$ & $0.91 \pm 0.11$ & $0.28 \pm 0.032$ \\
\hline$\Delta Z_{4}$ & \multicolumn{2}{|c|}{1.49} & \multicolumn{2}{|c|}{1.16} & \multicolumn{2}{|c|}{3.25} \\
\hline
\end{tabular}


Table 2. Operational characteristics of the technique of polarization-variable laser autofluorescence of histological sections of biopsy of cervix uteri endometrium of Group 1 (dysplasia) and Group 2 (cervical microinvasive carcinoma).

\begin{tabular}{lccc}
\hline$R(\%)$ & $I\left(\alpha_{0}=0^{\circ}\right)$ & $I\left(\alpha_{0}=90^{\circ}\right)$ & $I\left(\bar{\alpha}_{0}\right)$ \\
\hline$S e$ & 72 & 76 & 94 \\
$S p$ & 58 & 64 & 88 \\
$A c$ & 65 & 70 & 91 \\
\hline
\end{tabular}

autofluorescence proved to be efficient from the point of view of probative medicine ( $S e=94 \%$; $S p=88 \% ; A c=91 \%)$ in differential diagnostics of early stages types of cervix uteri cancer.

For the possible clinical application of the technique of polarization-variable laser autofluorescence, the tests "fluorescent cytology" within two statistically valid groups (confidence interval $p<0.001$ ) of samples mucous coat smears were

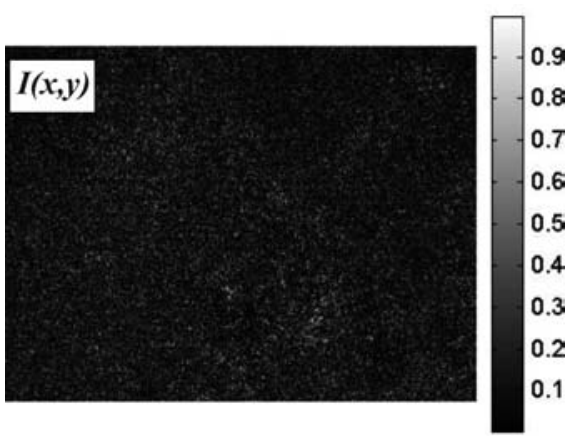

(a)

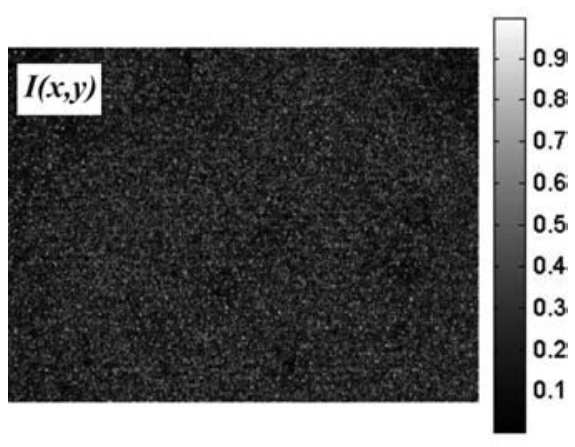

(c) performed. The obtained results for polarizationoptimal state of probing beam presented in Fig. 4.

The comparative analysis of the obtained autofluorescent images of cytological smears of both types (Fig. 4, parts (1) and (3)) revealed more intense luorescence of mucous coat porphyrins obtained from the surface of endometrium affected by the cervical microinvasive carcinoma. Quantitatively, this fact is illustrated by the histograms of distribution of fluorescence images intensities (Fig. 4, parts (2) and (4)). As it can be seen that for the sample of the group 2, the distribution $N(I)$ is characterized by bigger half-width and shifted extremum to the range of bigger values (Fig. 4, part (4)).

From the physical point of view, the obtained data can be connected with the influence of two factors. The first one, in Refs. 28-30, it is shown that autofluorescence in the red part of the spectrum is increasing together with the development of tumor. This phenomenon can be connected with the

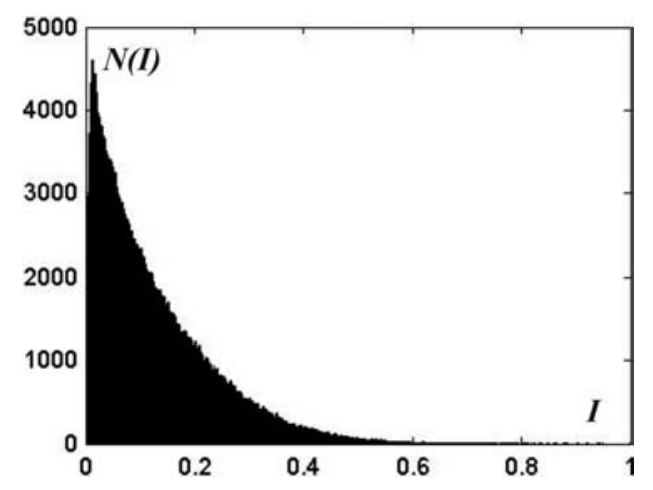

(b)

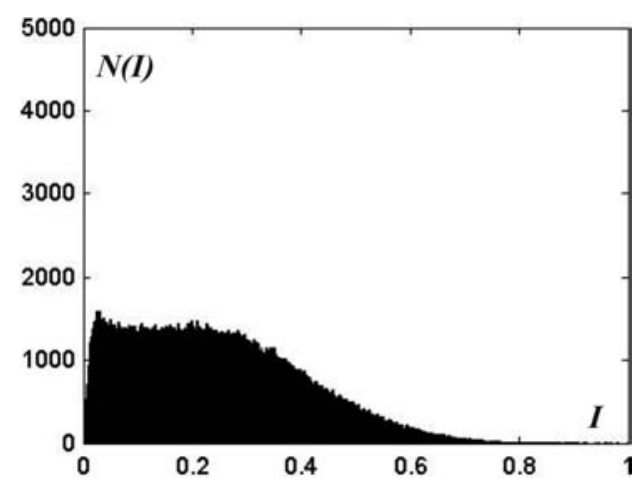

(d)

Fig. 4. Coordinate $(I(x, y))$ and statistical $(N(I))$ distributions of laser autofluorescence intensities of cytology smears of cervix uteri mucous coat in precancer state (parts (1), (2)) and cervical microinvasive carcinoma (parts (3), (4)) for the polarizationoptimal probing $\alpha_{0}=\bar{\alpha}_{0}$. Explanations are in the text. 
Table 3. Statistical $\left(Z_{i=1 ; 2 ; 3 ; 4}\right)$ parameters of distributions $I\left(\bar{\alpha}_{0}\right)$ of cytological smears of mucous coat of cervix uteri endometrium of Group 1 (dysplasia) and Group 2 (cervical microinvasive carcinoma).

\begin{tabular}{ccc}
\hline & \multicolumn{2}{c}{$I\left(\bar{\alpha}_{0}\right)$} \\
\cline { 2 - 3 }$Z_{i=1 ; 2 ; 3 ; 4}, \Delta Z_{i=1 ; 2 ; 3 ; 4}$ & Group 1 & Group 2 \\
\hline$Z_{1}$ & $0.11 \pm 0.018$ & $0.21 \pm 0.028$ \\
$\Delta Z_{1}$ & \multicolumn{2}{c}{1.9} \\
$Z_{2}$ & $0.13 \pm 0.019$ & $0.21 \pm 0.034$ \\
$\Delta Z_{2}$ & \multicolumn{2}{c}{1.62} \\
$Z_{3}$ & $0.54 \pm 0.087 \quad 0.83 \pm 0.12$ \\
$\Delta Z_{3}$ & \multicolumn{2}{c}{1.54} \\
$Z_{4}$ & $1.47 \pm 0.17$ & $0.81 \pm 0.11$ \\
$\Delta Z_{4}$ & \multicolumn{2}{c}{1.81} \\
\hline
\end{tabular}

increasing of the porphyrins concentration, which accumulate in the tissues of malignant new formations during the different stages of their development. The second one, malignant states are accompanied by the formation of birefringent liquid-crystal networks of porphyrins with the statistical formation of prevalent direction of optical axes $\rho^{*}$. Therefore, the conditions of polarization-optimal probing $\bar{\alpha}_{0}=\rho^{*}$ for such samples are realized more evidently in comparison with the samples of cervix uteri mucous coat endometrium with dysplasia.

Tables 3 and 4 present the averaged values and standard deviations of statistical moments $Z_{i=1 ; 2 ; 3 ; 4}(I)$ and operational characteristics of such a method.

The following quantitative (objective) criteria of diagnostics and differentiations of early forms of cervix uteri cancer are determined:

- the difference between the values of statistical moments of the first-fourth-orders characterizing the distributions $I\left(\bar{\alpha}_{0}\right)$ is for $Z_{1} \uparrow$ (by 1.9 times),

Table 4. Operational characteristics of the technique of polarization-variable laser autofluorescence of cytological smears of mucous coat of cervix uteri endometrium of Group 1 (dysplasia) and Group 2 (cervical microinvasive carcinoma).

\begin{tabular}{llll}
\hline$R$ & $S e$ & $S p$ & $A c$ \\
\hline$\%$ & 88 & 79 & 83 \\
\hline
\end{tabular}

for $Z_{2} \uparrow$ (by 1.62 times), for $Z_{3} \uparrow$ (by 1.54 times) and for $Z_{4} \downarrow$ (by 1.81 times).

Thus the method of statistical analysis of intensity distributions of laser polarization-variable autofluorescence proved to be efficient from the point of view of probative medicine $(S e=88 \%$; $S p=79 \% ; A c=83 \%)$ in differential diagnostics of early forms of cervix uteri cancer.

The usage of the method of polarization-variable autofluorescence provides:

- the substantial increasing of the specificity $(\sim 1.5$ times) of cytological screening;

- more validated collection of the biopsies of cervix uteri endometrium;

- the enhancement of the possibilities of diagnostics and differentiations of microfocal early forms of cervix uteri cancer.

\section{Conclusion}

(1) The optimal conditions of laser polarization fluorescence of liquid-crystal porphyrin networks were determined considering linear dichroism and birefringence of biological layers.

(2) The interconnections between the statistical moments characterizing laser autofluorescent images of histological sections of biopsy of cervix uteri endometrium and the peculiarities of its pathological states were found.

(3) Clinical efficiency of the technique of polarization-variable laser autofluorescence of liquidcrystal porphyrin networks of cytological smears of endometrium mucous coat in the task of diagnostics and differentiation of early forms of cervix uteri cancer is demonstrated.

\section{References}

1. S. Huard, Polarization of Light, J. Wiley and Sons, Ltd, New York (1997).

2. D. Goldstein, Polarized Light, 2nd Edition, Marcel Dekker, Inc., New York (2003).

3. M. H. Smith, P. Burke, A. Lompado, E. Tanner, L. W. Hillman, "Mueller matrix imaging polarimetry in dermatology," Proc. SPIE 3991, 210-216 (2000).

4. M. H. Smith "Interpreting Mueller matrix images of tissues," Proc. SPIE 4257, 82-89 (2001).

5. X. Wang, L. V. Wang "Propagation of polarized light in birefringent turbid media: A Monte Carlo study," J. Biomed. Opt. 7, 279-290 (2002). 
6. S. Lu, R. A. Chipman "Interpretation of Mueller matrices based on polar decomposition," J. Opt. Soc. Am. A 13, 1106-1113 (1996).

7. A. Pierangelo, A. Nazac, A. Benali, P. Validire, H. Cohen, T. Novikova, B. H. Ibrahim, S. Manhas, C. Fallet, M.-R. Antonelli, A.-D. Martino, "Polarimetric imaging of uterine cervix: A case study," Opt. Express 21, 14120-14130 (2013).

8. O. V. Angelsky, S. G. Hanson, A. P. Maksimyak, P. P. Maksimyak, "On the feasibility for determining the amplitude zeros in polychromatic fields," Optics Express 13, 4396-4405 (2005).

9. A. G. Ushenko, V. P. Pishak, "Laser polarimetry of biological tissue: Principles and applications," Handbook of Coherent-Domain Optical Methods: Biomedical Diagnostics, Environmental and Material Science, V. Tuchin eds., Vol. 1, pp. 93-138, Kluwer Academic Publishers (2004).

10. A. Y. Bekshaev, O. V. Angelsky, S. G. Hanson, C. Y. Zenkova, "Scattering of inhomogeneous circularly polarized optical field and mechanical manifestation of the internal energy flows," Phys. Rev. A 86, 023847 (2012).

11. Y. A. Ushenko, T. M. Boychuk, V. T. Bachynsky, O. P. Mincer, "Diagnostics of structure and physiological state of birefringent biological tissues: Statistical, correlation and topological approaches," Handbook of Coherent-Domain Optical Methods, pp. 107-148. Springer Science+Business Media New York (2013), ISBN 978-1-4614-5175-4.

12. Yu. A. Ushenko, G. B. Bodnar, G. D. Koval, "Classifying optical properties of surface-and bulkscattering biological layers with polarization singular states," J. Innov. Opt. Health Sci. 6, 1350018 (2013).

13. Y. A. Ushenko, "Investigation of formation and interrelations of polarization singular structure and Mueller-matrix images of biological tissues and diagnostics of their cancer changes," J. Biomed. Opt. 16(6), 066006 (2011).

14. Yu. A. Ushenko, "Statistical structure of polarization-inhomogeneous images of biotissues with different morphological structures," Ukr. J. Phys. Opt. 6, 63-70 (2005).

15. Yu. A. Ushenko, A. P. Peresunko, Bozan Adel Baku, "A new method of Mueller-matrix diagnostics and differentiation of early oncological changes of the skin derma," Adv. Opt. Technol. 2010, Article ID 952423 (2010).

16. Yu. A. Ushenko, O. I. Telenga, A. P. Peresunko, O. K. Numan, "New parameter for describing and analyzing the optical-anisotropic properties of biological tissues," J. Innov. Opt. Health Sci. 4, 463475 (2011).

17. Yu. A. Ushenko, "The feasibilities of using the statistical, fractal and singular processing of hominal blood plasma phase images during the diagnostics and differentiation of mammary gland pathological states," J. Innov. Opt. Health Sci. 5, 1150001 (2012).

18. S. Andersson-Engels, C. Klinteberg, K. Svanberg, S. Svanberg, "In vivo fluorescence imaging for tissue diagnostics," Phys. Med. Biol. 42, 815-824 (1997).

19. R. R. Alfano, B. B. Das, J. Cleary, R. Prudente, E. J. Celmer, "Light sheds light on cancer - Distinguishing malignant tumors from benign tissues and tumors," Bull N Y Acad Med. 67, 143-150 (1991).

20. M. Anidjar, D. Ettori, O. Cussenot, P. Meria, F. Desgrandchamps, A. Cortesse, P. Teillac, A. Le Duc, S. Avrillier, "Laser induced autofluorescence diagnosis of bladder tumors: Dependence on the excitation wavelength," J. Urol. 156, 1590-1596 (1996).

21. A. G. Bohorfoush, "Tissue spectroscopy for gastrointestinal diseases," Endoscopy 28, 372-380 (1996).

22. O. V. Angelskii, A. G. Ushenko, A. D. Archelyuk, S. B. Ermolenko, D. N. Burkovets "Structure of matrices for the transformation of laser radiation by biofractals," Quantum Electron. 29, 1074-1077 (1999).

23. R. Alfano, D. Tata, J. Cordero et al., "Laser induced fluorescence spectroscopy from native cancerous and normal tissue," IEEE Quantum Electron. 20, 15071511 (1984).

24. O. V. Angelsky, A. Ya. Bekshaev, P. P. Maksimyak, A. P. Maksimyak, I. I. Mokhun, S. G. Hanson, C. Yu. Zenkova, A. V. Tyurin, "Circular motion of particles suspended in a Gaussian beam with circular polarization validates the spin part of the internal energy flow," Opt. Express 20, 11351-11356 (2012).

25. J. Y. Qu, "Real time calibrated fluorescence imaging of tissue in vivo by using the combination of fluorescence and cross-polarized reflection," Biomedical Topical Meetings, Vol. 71 of OSA Trends in Optics and Photonics Series OSA, pp. 485-487, Washington, D.C. (2002).

26. N. Ghosh, S. K. Majumder, P. K. Gupta, "Polarized fluorescence spectroscopy of human tissues," Opt. Lett. 27, 2007-2009 (2002).

27. S. N. Savenkov, V. V. Marienko, E. A. Oberemok, O. I. Sydoruk, "Generalized matrix equivalence theorem for polarization theory," Phys. Rev. E. 74, 605-607 (2006).

28. R. Stroka, R. Baumgartner, A. Buser, C. Ell, D. Jocham, E. Unsold, "Laser assisted detection of endogenous porphyrin in malignant diseases," SPIE Proc. 1641, 99-105 (1991).

29. M.-A. D'Hallewin, A. R. Kamuhabwa, T. Roskams, P. A. M. De Witte, L. Baert, "Hypericin-based fluorescence diagnosis of bladder carcinoma," BJU Int. 89, 760-763 (2002).

30. M. A. D'Hallewin, L. Bezdetnaya, F. Guillemin, "Fluorescence detection of bladder cancer: A review," Eur. Urol. 42, 417-425 (2002). 